\title{
Bases on Multipunctured Riemann Surfaces and Interacting Strings Amplitudes
}

\author{
V. A. Sadov \\ L.D. Landau Institut for Theoretical Physics, Kosygina 2, SU-117940 GSP-1, Moscow V-334, \\ USSR
}

Received January 15, 1990

\begin{abstract}
The Krichever-Novikov bases are studied on Riemann surfaces with more-than-two punctures. The bases are presented and the completness theorem is proven for the case of integer (up to a common constant) momenta. Then the interacting strings are considered, the amplitudes and partition functions are obtained, comparable with that of path-integral approach. For the amplitudes the simple geometric implication is proposed.
\end{abstract}

\section{Introduction}

The works $[1,2]$ of Novikov and Krichever stimulated an interest in the global operator expansions on Riemann surfaces of arbitrary genus. Originally KN considered the bases on these surfaces with two marked points. Physically it corresponds to a single self-interacting string at the perturbation theory order, equal to genus. In $[8,9]$ some attempts are presented to generalise the constructions of $[1,2]$ to the multi-point case. The work [10] concerns representations of Heisenberg-type $\mathrm{KN}$ algebra.

In their recent articles [3, 4] Krichever and Novikov applied the bases of [1, 2] to the operator quantization of the self-interacting string to obtain the amplitude and the partition function. The canonical operator product expansions of CFT were rederived using the well-defined quantities.

Our main goal in this paper is to generalise the results of [1-4] to obtain the multistrings quantization. We will get the correct tensor bases, the quantum state vectors and, finally, the scattering amplitudes, which turn out to be the generalised Kobo-Nielsen ones, obtained earlier in Polyakov's theory [7]. It is proven thus that the Krichever-Novikov operator quantization does leads to correct effects.

Moreover, this approach makes more transparent the geometric essence of bosonic amplitudes. These are simply related to the "Euclidean time" coordinates, intrinsic to $\mathrm{KN}$ theory. 


\section{Krichever-Novikov Bases for Multipoint Diagrams}

There is a well known way to describe interacting strings at given perturbation theory order. Riemann surface $\Gamma$ (see Appendix A for the notation) with marked points $Q_{i}$ endowed with real numbers $p_{i}$ presents the world sheet for in- and outgoing strings, attached to $\Gamma$ in $Q_{i}$. We divide these points in two sets - "in": $\left\{Q_{i} \mid p_{i}>0\right\}$; "out": $\left\{Q_{i} \mid p_{i}<0\right\}$. The set of data $\left\{\Gamma, Q_{i}, p_{i}\right\}$ we call a "diagram."

On $\Gamma$, there is a special 1 -form $d k$ [1], that defines the "Euclidean time."

This differential enjoys the following properties:

a) it is holomorphic on $\Gamma$ except for $Q_{i}$, where it has simple poles with residues $p_{i}$, b) it is normalised by the condition $\operatorname{Re} \oint_{\gamma} d k=0, \forall \gamma \in H_{1}\left(\Gamma \backslash Q_{i}\right)$.

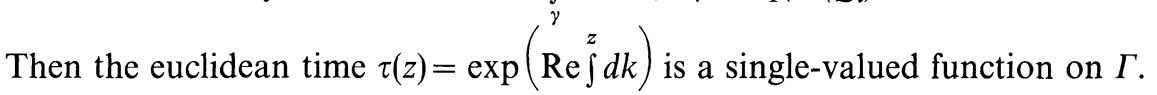
The level lines of this function define the contours set $C_{r}$. The set produces a fibration of $\Gamma$ which may be thought of as a picture of interacting strings which varies in time.

The operator quantization uses Fourier-type bases on $C_{\tau}$. Note, that $C_{\tau}$ and therefore, bases set essentially depend on momenta $p_{i}$. This paper concerns the case all the momenta being integer - up to a common factor, i.e. $\frac{p_{i}}{p_{j}}$ being rational. It is possible to pick the appropriate units to make $p_{i}$ be integers in fact. set.

Introduce an important value $l=\sum_{i \in i n} p_{i}$ - the total (integer) momentum of "in"

Riemann-Roch theorem implies, that for every $\lambda \neq 0,1$ and every integer $n$ there are $l$ independent tensor fields $f_{n \alpha}^{\lambda}(z)$ of weight $\lambda$ such that:

a) they are holomorphic on $\Gamma$, except, possibly, for $Q_{i}$,

b) in the vicinities of $Q_{i}$ in local coordinates $z_{i}$ they take the form:

$$
f_{n \alpha}^{\lambda}(z)=\phi_{n, \alpha}^{i} z_{i}^{p_{i} n-S_{i}(\lambda, g)}\left(1+o\left(z_{i}\right)\right)(d z)^{\lambda},
$$

where $S_{i}(\lambda, g)$ are arbitrary integers, $\sum_{i} s_{i}=(2 \lambda-1)(g-1)+l$.

For the exceptional cases $\lambda=0,1$ the bases also exist, their local form being slightly different.

Theorem 1. Let $C_{\tau}$ be non-degenerate, $f^{\lambda}(\sigma)$-be any smooth tensor field on $C_{\tau}$, then

$$
f^{\lambda}(\sigma)=\sum_{n} \sum_{\alpha=1}^{l} f_{n \alpha}^{\lambda}(\sigma) \frac{1}{2 \pi i} \oint_{C_{\tau}} f^{\lambda}(\sigma) f_{-n \alpha}^{1-\lambda}(\sigma),
$$

where $S_{i}(\lambda, g)$ are so chosen, that $S_{i}(1-\lambda, g)+S_{i}(\lambda, g)=1$, and

$$
\oint_{C_{\tau}} f_{n, \alpha}^{\lambda}(\sigma) f_{m, \beta}^{1-\lambda}(\sigma)=\delta_{n,-m} \delta_{\alpha \beta} .
$$

Proof. Consider first the simplest multipoint diagram - that one with all the momenta being the same: $\left|p_{i}\right|=\left|p_{j}\right|$. (The case first considered in [4]). By the proper rescaling one can set $\left|p_{i}\right|=1$. For this special case it is convenient to change slightly our notation. There are $l$ points in "in" set and $l$ points in "out" set, so we denote "in" points as $Q_{+\alpha}$ and "out" points as $Q_{-\alpha}, \alpha$ runs from 1 to $l$. Essentially, apart from being easy to handle with, this case is the generic one for the general case. 
Specifying the diagram, the powerful soliton theory technique of $\mathrm{KN}$ [1] is applied. In addition to the "physical" vertices $Q_{ \pm \alpha}$ fix $g+l-1$ point divisor $Z=\sum_{i=1}^{g+l-1} \gamma_{i}$. There is a 1-differential $d \Omega$ such that:

a) it is holomorphic on $\Gamma$, except for $Q_{ \pm \alpha}$, where it has simple poles of residues $p_{i}(= \pm 1)$,

b) it has zeroes in $Z$.

Besides, $d \Omega$ has $g+l-1$ zeros in $Z_{+}=\sum_{i=1}^{g+l-1} \gamma_{i}^{+}$. For any integer $n$ consider two $l$-dimensional spaces produced by the functions $\psi_{n}^{\alpha}(z)$ and $\psi_{n}^{\alpha+}(z)$. These functions have poles $\operatorname{divisors}$ such that $\operatorname{div}\left(\psi_{n}^{\alpha}\right) \geqq-Z, \operatorname{div}\left(\psi_{n}^{\alpha+}\right) \geqq-Z_{+}$, and specified by their local expansion near $Q_{i}$ :

$$
\begin{gathered}
\psi_{n}^{\alpha}\left(z_{i}\right)=c_{n}^{\alpha} z^{p_{i} n}\left(1+o\left(z_{i}\right)\right), \\
\psi_{n}^{\alpha+}\left(z_{i}\right)=c_{n}^{\alpha+} z^{-p_{i} n}\left(1+o\left(z_{i}\right)\right)
\end{gathered}
$$

$\left|p_{i}\right|=1$, index $\alpha$ marks subspaces elements, $1<\alpha<l$.

A)

There are two useful bases of $\psi_{n}^{\alpha}$ and $\psi_{n}^{\alpha+}$,

$$
\begin{gathered}
\psi_{n}^{\alpha}\left(z_{-\beta}\right)=\frac{\delta_{\alpha \beta}}{c_{\alpha-\beta}^{+}} z_{-\beta}^{-n}\left(1+o\left(z_{-\beta}\right)\right), \\
\psi_{n}^{\alpha}\left(z_{+\beta}\right)=c_{\alpha+\beta} z_{+\beta}^{n}\left(1+o\left(z_{+\beta}\right)\right), \\
\psi_{n}^{+\alpha}\left(z_{-\beta}\right)=c_{\alpha-\beta}^{+} z_{-\beta}^{n}\left(1+o\left(z_{-\beta}\right)\right), \\
\psi_{n}^{+\alpha}\left(z_{+\beta}\right)=\frac{\delta_{\alpha \beta}}{c_{\alpha+\beta}} z_{+\beta}^{-n}\left(1+o\left(z_{+\beta}\right)\right) .
\end{gathered}
$$

Discuss it briefly. There is no problem to "diagonalise" $\psi_{n}^{\alpha}(z)$ and $\psi_{n}^{+\alpha}(z)$ to leave the highest $\left(n^{\text {th }}\right)$ order poles of $\psi_{n}^{\alpha}(z)\left(\psi_{n}^{+\alpha}(z)\right)$ only on $Q_{+\alpha}\left(Q_{-\alpha}\right)$. Doing this, fix $\alpha$ and consider $\psi_{n}^{+\alpha}(z) \psi_{n}^{\alpha}(z) d \Omega$. This 1 -differential has two simple poles in $Q_{+\alpha}$ and $Q_{-\alpha}$ with residues $c_{+\alpha}^{\alpha} c_{+\alpha}^{+, \alpha}$ and $-c_{-\alpha}^{\alpha} c_{-\alpha}^{+, \alpha}$, their sum being zero. This remarkable identity enables us to normalise as above. We like this basis for

Proposition 1.

$$
d \Omega=\frac{d k}{\sum_{\alpha=1} l\left\langle\psi_{n}^{+\alpha} \psi_{n}^{\alpha}\right\rangle_{n}}
$$

$\langle\cdot\rangle_{n}$ here stands for the average on $n$. Theta-functional formulae for $\psi_{n}^{+\alpha}$ and $\psi_{n}^{\alpha}$ imply that the product $\psi_{n}^{+\alpha} \psi_{n}^{\alpha}$ is quasiperiodic as a function of $n$, so the average is sensable. From the definition of $d \Omega$ and (1.4), it follows that

$$
\frac{1}{2 \pi i} \oint_{C_{\tau}} \psi_{m}^{+\beta}(\sigma) \frac{d k}{\sum_{\alpha=1}^{l}\left\langle\psi_{n}^{+\alpha} \psi_{n}^{\alpha}\right\rangle_{n}}=\delta_{n 0} .
$$

B) Whereas $\mathrm{A}$ is distinguished for (1.4) and (1.5), the most convenient are orthogonal bases, where $\oint_{\mathcal{C}_{\tau}} \psi_{n}^{\alpha} \psi_{m}^{+, \beta} d \Omega=2 \pi i \delta_{\alpha \beta} \delta_{m n}$. One can get these bases through the usual orthogonalisation procedure. Both $\mathrm{A}$ and $\mathrm{B}$ bases sets being nondegenerated, the matrix $M_{\alpha \beta}^{n}$ is defined, that transforms one into the other. Fix now orthogonal bases $\psi_{n}^{\alpha}, \psi_{n}^{+, \alpha}$. 
Lemma. For every non-singular $C_{\tau}$ every smooth function $F(\sigma)$ on $C_{\tau}$ has an expansion:

$$
F(\sigma)=\sum_{n} \psi_{n}^{\beta}(\sigma) \frac{1}{2 \pi i} \oint_{C_{\tau}} F\left(\sigma^{\prime}\right) \psi_{m}^{+\beta}\left(\sigma^{\prime}\right) \frac{d k\left(\sigma^{\prime}\right)}{\sum_{\alpha=1}^{l}\left\langle M_{\alpha \beta}^{n} \psi_{n}^{+\alpha} \psi_{n}^{\alpha}\right\rangle_{n}} .
$$

Proof. Let $S_{N}(\sigma)$ be a partial sum of (1.6), then

$$
\begin{aligned}
S_{N}(\sigma)-F(\sigma)= & \sum_{n=-N}^{N} \frac{1}{2 \pi i} \oint_{C_{\tau}}\left(F\left(\sigma^{\prime}\right)-F(\sigma)\right) \psi_{n}^{\beta}(\sigma) \psi_{m}^{+\beta}\left(\sigma^{\prime}\right) \\
& \times \frac{d k\left(\sigma^{\prime}\right)}{\sum_{\alpha=1}^{l}\left\langle M_{\alpha \beta}^{n} \psi_{n}^{+\alpha} \psi_{n}^{\alpha}\right\rangle_{n}} .
\end{aligned}
$$

Choose some function $\lambda(Q)$ on $\Gamma$ with $g+1$ poles in $Q_{-\alpha}$ 's and a zero in $Q_{+\alpha}$ 's.

\section{Proposition 2.}

$$
\begin{gathered}
\lambda(z) \psi_{n}^{\alpha}(z)=\sum_{i=1}^{g+1} h_{\beta i}^{\alpha n} \psi_{n+i}^{\beta}(z), \\
\lambda(z) \psi_{n}^{+\alpha}(z)=\sum_{i=1}^{g+1} h_{\alpha i}^{\beta n-i} \psi_{n-i}^{+\beta}(z) .
\end{gathered}
$$

(The equations of that kind first appeared in [6].) There are two facts stated:

1) The right-hand side of (1.8) contains only $g$ terms of the kind $\psi_{n}^{\alpha}(z)$-it follows from the count of the number of zeros and poles in the left-hand side,

2) coefficients $h_{\beta j}^{\alpha i}$ in the right-hand side of (1.8.1) and (1.8.2) are the same because of

$$
h_{\beta j}^{\alpha i}=\frac{1}{2 \pi i} \oint_{C_{\tau}} \lambda(\sigma) \psi_{n}^{\alpha}(\sigma) \psi_{n+i}^{+\beta}\left(\sigma^{\prime}\right) d \Omega
$$

By (1.8.1) and (1.8.2) one gets

$$
\begin{aligned}
\sum_{n=-N}^{N} \psi_{n}^{\alpha}(\sigma) \psi_{n}^{+\alpha}\left(\sigma^{\prime}\right)= & \sum_{i=1}^{g+1}\left(\sum_{n=N-i}^{N} h_{\beta i}^{\alpha n} \psi_{n+i}^{\beta}(z) \psi_{n}^{+\alpha}\left(\sigma^{\prime}\right)\right. \\
& \left.-\sum_{n=-N-i} h_{\beta i}^{\alpha n} \psi_{n+i}^{\beta}(z) \psi_{n}^{+\alpha}\left(\sigma^{\prime}\right)\right)
\end{aligned}
$$

The Dirichlet kernel analogue

$$
D(\sigma)=\sum_{n=-N}^{N} \frac{\psi_{n}^{\beta}(\sigma) \psi_{n}^{+\beta}\left(\sigma^{\prime}\right)}{\sum_{\alpha=1}^{l}\left\langle M_{\alpha \beta}^{n} \psi_{n}^{+\alpha} \psi_{n}^{\alpha}\right\rangle_{n}}=\Phi_{N} e^{(N-g+1) k(\sigma)}+\Phi_{-N} e^{-N k(\sigma)}
$$

oscillates quickly as $N \rightarrow \infty$.In a usual way it implies that $S_{N}(\sigma)-F(\sigma) \rightarrow 0(N \rightarrow \infty)$.

The lemma is proven.

We are in a position now to make use of $\psi_{n}^{\alpha}$ to obtain all the tensor bases (1.0) and to prove Theorem 1 for the special case $\left|p_{i}\right|=1$. Fix $f_{0}^{\lambda}(z)$. It has $g+l-1$ zeroes divisor $Z$. Find $\psi_{n}^{\alpha}$ and $\psi_{n}^{+\alpha}$ out of $Z$ as prescribed above and put $f_{n, \alpha}^{\lambda}(z)=f_{0}^{\lambda}(z) \psi_{n}^{\alpha}(z)$. 
So defined, $f_{n(z), \alpha}^{\lambda}$ does satisfy all the properties the basic field (1.0) should. As $\psi_{n}^{\alpha}$ basis is full, $f_{n(z), \alpha}^{\lambda}$ bases are also full on the contours $C_{\tau}$ for all $\lambda$; Theorem 1 is thus proven.

Return to the general case of "in" state including $N_{\text {in }}$ points $Q_{1}, \ldots, Q_{N_{\text {in }}}$ "out" state including $N_{\text {out }}$ points $Q_{N_{\text {in }}+1}, \ldots, Q_{N_{\text {in }}+N_{\text {out }}}$ of integer momenta $p_{i}$. Let $l$ be the total momentum of the "in" state: $l=\sum_{\text {in }} p_{i}$.

Note now that this general picture can be obtained out of a special one, treated above. Really, take an auxilliary diagram with $l$ "in" and $l$ "out" points of momenta \pm 1 , respectively. We shall get a point of (positive, to say) momentum $p$ on fusing together $p$ "in" points of the auxilliary diagram. While fusing, we should be on guard not to spoil the contours set and the bases $f_{n}^{\lambda, \alpha}(z)$. It is obvious, however, that a given contour is not influenced as much by the details of fusion, providing the size of the fusing system is small enough. Thus the contours set is continuous. As for the bases sets $\psi_{n}^{\alpha}$ and $\psi_{n}^{+\alpha}$ of the lemma (and, therefore, all tensor bases) more care is required.

Let us fuse two points $Q_{-1}$ and $Q_{-2}$ of the "out" set.

Proposition 3. In fusing, one of the points of $Z_{+}$is being sandwiched between $Q_{-1}$ and $Q_{-2}$.

Proof. Take $Q_{-1}$ and $Q_{-2}$ close enough to be on the same coordinate map, then

$$
d \Omega=\left(\frac{-1}{z_{-1}}+\frac{-1}{z_{-1}-u}+o(1)\right) d z_{-1} .
$$

It is written through $Q_{-1}$ local coordinate $z_{-1}$, so $u$-it is a $Q_{-2}$ 's coordinate on the map - is a fusion parameter. It is easy to check that $d \Omega$ has a zero at the point $\frac{u}{2}+o\left(u^{2}\right)$ that belongs to $Z_{+}$by definition.

The proposition claims that on fusing $p$ "in" ("out") points $Q_{i}$ to the one $Q$, also $p \psi_{n}^{\alpha}$ s $\left(\psi_{n}^{+\alpha}\right.$ s) poles join $Q, \operatorname{deg}(Z),(\operatorname{deg}(Z+))$ being the same (and equal to $\left.l\right)$. Thus the dimensions of $\psi_{n}^{\alpha}$ bases do not change, i.e. there is no degeneration. Consider the basis elements fusion in more details.

In the $Q_{-1}$ local map of Proposition 3 above we have

$$
\psi_{n}^{+\alpha}=c^{\alpha} z^{-n}(z-u)^{-n}\left(A+\frac{B}{z-\frac{u}{2}+o\left(u^{2}\right)}\right) \rightarrow \text { const } \cdot z^{-2 n-1}(1+o(z)) .
$$

Although the leading power $2 n+1$ changes by 2 , due to two independent coefficients in the left-hand side of (1.13) there are two functions with such asymptotics, the gap does not appear. The "fused" bases of the lemma take the form:

$$
\begin{gathered}
\psi_{n}^{+\alpha}=c_{i}^{+\alpha} z_{i}^{n p_{i}-\left(p_{i}-1\right) \varepsilon_{i}}\left(1+o\left(z_{i}\right)\right), \\
\psi_{n}^{\alpha}=c_{i}^{\alpha} z_{i}^{n p_{i}-\left(p_{i}-1\right)\left(1-\varepsilon_{i}\right)}\left(1+o\left(z_{i}\right)\right),
\end{gathered}
$$

where $\varepsilon_{i}$ is one for "out" points and zero for "in" points and the tensor bases take the form (1.0).

Noting that the expressions (1.7-1.11) of the lemma are continuous on the fusion parameter, Theorem 1 is proven for the case of arbitrary (integer up to a constant) momenta. 
Remark 1. Some explanations should be given on the choice of $l$, for the global rescalings group gives rise to equivalence of the physically inequivalent diagrams. Thus, all the diagrams with two marked points of [1-3] belong to the same equivalence class. As the contours set and, consequently, the bases should depend on the class only, there is a way to "reduce" $l$ in terms of bases re-numeration. Namely, for the set $\left\{\Gamma, Q_{i}, 2 p_{i}\right\}$ at each $n$ the $2 l$-dimensional space of independent basis tensors reduces to two $l$-dimensional ones. In the first subspace $2 n p_{i}-S_{i}$ $\leqq \operatorname{deg}\left(Q_{i}\right) \leqq 2 n p_{i}+p_{i}-1-S_{i}$, while in the second $2 n p_{i}+p_{i}-S_{i} \leqq \operatorname{deg}\left(Q_{i}\right) \leqq 2(n+1) p_{i}$ $-1-S_{i}$. Re-numerating them to be $2 n^{\text {th }}$ and $2 n+1^{\text {th }}$, we arrive at the bases of the diagram $\Gamma, Q_{i}, p_{i}$.

Remark 2. The bases fields are uniquely determined by the condition (1.0). For later references, however, present the explicit formulae through the prime-form and Fay's $\sigma$-differential (A.4-A.6),

$$
\begin{aligned}
f_{n}^{\lambda}(z)= & \prod_{i=1}^{N_{\text {in }}+N_{\text {out }}} E^{n_{i}}\left(z, Q_{i}\right) \sigma(z)^{2 \lambda-1}\left[\sum_{i=1}^{N_{\text {in }}} \frac{\Theta[\varrho]\left(z-Q_{i}+U\right)}{E\left(z, Q_{i}\right)}\right. \\
& \left.\times\left(C_{i}^{0}+C_{i}^{1} \partial_{Q_{i}} \log \frac{\Theta[\varrho]\left(z-Q_{i}+U\right)}{E^{n_{i}}\left(z, Q_{i}\right)}+\sum_{j=2}^{p_{i}-1} C_{i}^{j} \partial_{Q_{i}}^{j} \log E\left(z, Q_{i}\right)\right)\right]
\end{aligned}
$$

where $n_{i}=n p_{i}-s_{i}, \quad \sum_{i} s_{i}=(1-2 \lambda)(g-1)-1, \quad U=\sum_{i} p_{i} Q_{i}-(2 \lambda-1) \Delta$ and $C_{i}^{j}$ are coordinates in the $l\left(=\sum_{\text {in }} p_{i}\right)$-dimensional space. The theta-functional characteristic $\varrho$ specifies the boundary conditions on the cut $\Gamma$. Exceptional cases $\lambda=0,1$ can be handled in the same spirit.

\section{The Applications to the Operator Quantum Theory of Interacting Strings}

The vector fields $e_{n}^{\alpha}=f_{n}^{(-1) \alpha}$ generate the almost graded $\mathrm{KN}$ algebra:

$$
\left[e_{n}^{\alpha}, e_{m}^{\beta}\right]=\sum_{\substack{1 \leqq \gamma \\ L_{1} \leqq k \leqq L_{2}}} c_{k \alpha \beta}^{n m \gamma} e_{n+m-k}^{\gamma} .
$$

The limits $L_{1}$ and $L_{2}$ depend on the choice of $S_{i}(-1, g)$ but are finite. Tensor fields $f_{n}^{(\lambda) \alpha}$ for each $\lambda$ are endowed with KN module properties:

$$
e_{n}^{\alpha} f_{m}^{(\lambda) \beta}=\sum_{\substack{1 \leqq \gamma \leqq l \\ M_{1} \leqq k \leqq M_{2}}} r_{k \alpha \beta}^{n m \gamma} f_{n+m-k}^{(\lambda) \gamma} .
$$

In quantum theory there appear central extensions of 1-forms and vector fields algebras - these are the Heisenberg algebra and Virasoro algebra. The first is generated by the elements $\alpha_{n}^{\alpha}$ and $t$ with the commutation relations

$$
\begin{gathered}
{\left[\alpha_{n}^{\alpha}, \alpha_{m}^{\beta}\right]=\gamma_{n m} t,} \\
{\left[\alpha_{n}^{\beta}, t\right]=0,} \\
\gamma_{n m}=\frac{1}{2 \pi i} \oint_{C_{\tau}} f_{n}^{1, \alpha} d f_{m}^{1, \beta} .
\end{gathered}
$$


In turn, the Virasoro algebra is generated by $e_{n}^{\alpha}$ and $t$ and looks like the

$$
\begin{gathered}
{\left[e_{n}^{\alpha}, e_{m}^{\beta}\right]=\sum_{\substack{1 \leq \gamma \leq l \\
L_{1} \leqq k \leqq L_{2} \\
\left[e_{n}^{\alpha}, t\right]=0 ;}} c_{k \alpha \beta}^{n m y} e_{n+m-k}^{\gamma}+\chi_{n m} t,} \\
{\left[e^{\alpha},\right.}
\end{gathered}
$$

the cocycle $\chi_{n m}$ is the standard Gelfand-Fuchs' one on the contours $C_{\tau}$. As in the two-point case the cocycle is local - it equals zero identically outside a strip of the finite width around the diagonal $|n+m|=0$.

Verma moduli analogues are constructed out of semi-infinite wedge products of basic $\lambda$-forms. The characteristic representative looks like:

$$
f_{n_{1}}^{\lambda, \alpha_{1}} \wedge f_{n_{2}}^{\lambda, \alpha_{2}} \wedge \ldots \wedge f_{n_{k}}^{\lambda, \alpha_{k}} \bigwedge_{\substack{m>n_{k} \\ 1 \leqq \alpha \leqq l}} f_{m}^{\lambda, \alpha}
$$

- the right form

$$
\bigwedge_{\substack{m<n_{k} \\ 1 \leqq \alpha \leqq l}} f_{m}^{\lambda, \alpha} \wedge f_{n_{k}}^{\lambda, \alpha_{k}} \wedge \ldots \wedge f_{n_{2}}^{\lambda, \alpha_{2}} \wedge f_{n_{1}}^{\lambda, \alpha_{1}}
$$

- the left form, $n_{1} \leqq \ldots \leqq n_{k}$. The right KN moduli are used for "in" states, the left for "out" states.

To get the multistring state vectors, following the arguments of [3], consider the case $\lambda=\frac{1}{2}$. When handling $\frac{1}{2}$-forms an additional structure appears - it is the global boundary conditions on the cut $\Gamma$. Take the unitary representation of the fundamental group $\pi_{1}(\Gamma) \varrho: \pi_{1}(\Gamma) \rightarrow S^{1}$. In the canonical 1-cycles basis $a_{i}, b_{i}$ it is defined by $2 g$ real parameters $\varrho_{1}, \ldots, \varrho_{2 g}$ :

$$
\varrho\left(a_{j}\right)=\exp \left(2 \pi i \varrho_{j}\right), \quad \varrho\left(b_{j}\right)=\exp \left(2 \pi i \varrho_{j+g}\right) .
$$

The vector $\varrho$ is a theta-functional characteristic of the explicit formula (1.15). The case when $\varrho$ is a half-period corresponds to the "ordinary" spin structures.

Thus "in", "out" vectors are:

$$
\mid \text { in }\rangle=\bigwedge_{\substack{n \geqq 3 / 2 \\ 1 \leq \alpha \leq l \\ 1 \leqq i \leqq N_{i} n}} \Phi_{n}^{\alpha}\left(z_{i}\right),
$$

Now consider $\mid$ in $\rangle$, |out $\rangle$ in more detail. From (1.0), near $Q_{i}$

$$
\Phi_{n}^{\alpha}\left(z_{i}\right)=z^{n p_{i}-\frac{p_{i}}{2}} \sum_{s=0}^{\infty} \xi_{s}^{\alpha i}(n) z_{i}^{s} .
$$

Substituting this expansion for $\Phi_{n}^{\alpha}\left(z_{i}\right)$ into (2.9) one gets:

$$
\mid \text { in }\rangle=\bigwedge_{n \geqq 3 / 2} z^{n p_{i}-\frac{p_{i}}{2}} \sum_{s=0}^{p_{i}-1} \xi_{s}^{\alpha_{i}}(n) z_{i}^{\mathrm{s}} .
$$


For each $\Phi_{n}^{\alpha}\left(z_{i}\right)$ only $p_{i}$ first terms of expansion survive; the other one is killed by $\Phi_{n+1}^{\alpha}\left(z_{i}\right)$.

After all,

$$
\begin{aligned}
\quad \mid \text { in }\rangle=\bigwedge_{n \geqq 3 / 2} Z_{n}^{\text {in }}, \\
\quad \times \operatorname{det}\left(\begin{array}{ccccc}
\text { in } & \bigwedge_{1 \leqq i \leqq N_{\text {in }}} z_{i}^{n p_{i}-\frac{\left|p_{i}\right| 2}{2}} \wedge \ldots \wedge z_{i}^{(n+1) p_{i}-\frac{\left|p_{i}\right|}{2}-1} \\
\xi_{01}^{11}(n) & \ldots & \xi_{p_{1}-1}^{11}(n) & \xi_{0}^{12}(n) & \ldots \\
\vdots & \ddots & \vdots & \vdots & \ddots \\
\xi_{0}^{l 1}(n) & \ldots & \xi_{p_{1}-1}^{l 1}(n) & \xi_{0}^{l 2}(n) & \ldots
\end{array}\right) .
\end{aligned}
$$

Choose "in" normalisation - take $\mid$ in $\rangle$ equals the "in" vector of free strings:

$$
\mid \text { in }\rangle=z_{1}^{p_{1}} \wedge z_{2}^{p_{2}} \wedge \ldots \wedge z_{N_{\text {in }}}^{p_{N_{\text {in }}}} \wedge z_{1}^{p_{1}+1} \wedge \ldots
$$

In the normalisation proposed, the determinant in the right-hand side of (2.11) equals one, the <out $\mid$ vector being

$$
\begin{gathered}
\langle\text { out }|=\bigwedge_{n \leqq 3 / 2} Z_{n}^{\text {out }} \\
Z_{n}^{\text {out }}=\bigwedge_{N_{\text {in }} \leqq i \leqq N_{\text {in }}+N_{\text {out }}} z_{i}^{n p_{i}-\frac{\left|p_{i}\right|}{2}} \wedge \ldots \wedge z_{i}^{(n+1) p_{i}-\frac{\left|p_{i}\right|}{2}-1} \\
\times \operatorname{det}\left(\begin{array}{ccccc}
\xi_{0}^{1 N_{i} n}(n) & \ldots & \xi_{p_{1}}^{1 N_{i} n 1}(n) & \xi_{0}^{1 N_{i} n+1}(n) & \ldots \\
\vdots & \ddots & \vdots & \vdots & \ddots \\
\xi_{0}^{l N_{i} n}(n) & \ldots & \xi_{p_{1}-1}^{l N_{i} n}(n) & \xi_{0}^{l N_{i} n+1}(n) & \ldots
\end{array}\right) .
\end{gathered}
$$

The vacua "in", "out" states are determined as

$$
\begin{gathered}
|0\rangle=\bigwedge_{n \geqq 1 / 2} Z_{n}^{\text {in }}=1 \wedge z_{1} \wedge z_{2} \wedge \ldots, \\
\langle 0|=\bigwedge_{n \leqq 1 / 2} Z_{n}^{\text {out }}
\end{gathered}
$$

the "in" vacuum coincides with the free strings.

The amplitude is defined as $A\left(p_{1}, \ldots, p_{k}\right)=\frac{\langle\text { out }| \text { in }\rangle}{\langle 0 \mid 0\rangle}$ and from $(2.12-2.14)$ its (regularized) value is

whereas the partition function

$$
A\left(p_{1}, \ldots, p_{k}\right)=\frac{1}{Z_{1 / 2}^{\text {out }}}
$$

$$
Z_{F}=\langle 0 \mid 0\rangle \text {. }
$$

The internal product $\langle\cdot\rangle$ is introduced here as in [3]. Namely, for the left-infinite form $f(2.5)$ and the right-infinite form $g(2.4)$ consider the wedge product $f \wedge g$. If this infinite in both directions form coincides with some $\sigma$-permutation of the standard one $\langle$ out $|$ in $\rangle$, then we define $\langle f \mid g\rangle=\operatorname{sign}(\sigma)$. In all other cases the 
product $\langle f \mid g\rangle$ is set to be zero by definition. On the linear combinations of the basic forms $(2.4-2.5)$ the product $\langle\cdot\rangle$ is introduced by linearity both in the first and the second arguments.

Although very natural, that definition has an important physical implication. Let us introduce the "Euclidean time" coordinates $\xi_{i}$ in the vicinities of $Q_{i}$ through a 1 -form, $d k$

$$
\begin{gathered}
d k=d \log \prod_{i=1}^{k} E^{p_{i}}\left(z, Q_{i}\right)-2 \pi i \sum_{i, j=1}^{g}(\operatorname{Im} \tau)_{i j}^{-1}\left(\operatorname{Im} \sum_{k} p_{k} Q_{k}\right)_{i} \omega_{j}(z), \\
\xi_{i}=\exp \frac{1}{p_{i}} k\left(z_{i}\right) .
\end{gathered}
$$

Theorem 2. Written in the Euclidean time coordinates, the amplitude $A\left(p_{1}, \ldots, p_{K}\right)$ equals one identically.

Proof. To prove the theorem and thus to establish the operator quantisation approach of Novikov and Krichever let us calculate the amplitude explicitly. For technical reasons it is convenient to obtain it directly from (2.13-2.15) just for the basic case $p_{i}= \pm 1$ and then make use of the ordinary free bosonic theory fusion rules.

The basic $\frac{1}{2}$-differentials are:

$$
\Phi_{n}^{\beta}=\prod_{\alpha=1}^{l}\left[\frac{E\left(z, Q_{+\alpha}\right)}{E\left(z, Q_{-\alpha}\right)}\right]^{n+1 / 2} \sum_{\alpha=1}^{l} C_{\alpha}^{\beta}(n) \frac{\Theta[\varrho]\left(z-Q_{+\alpha}+\left(n+\frac{1}{2}\right) U\right)}{E\left(z, Q_{+\alpha}\right)},
$$

$U=\sum_{\alpha=1}^{l} Q_{+\alpha}-Q_{-\alpha}$

"In" normalisation gives:

$$
\operatorname{det} C^{-1}=\prod_{\alpha \neq \beta}\left(\frac{E\left(Q_{+\alpha}, Q_{+\beta}\right)}{E\left(Q_{+\alpha}, Q_{-\beta}\right)}\right)^{n+1 / 2} \prod_{\alpha} E^{-n-1 / 2}\left(Q_{+\alpha}, Q_{-\alpha}\right) \Theta^{l}[\varrho]\left(\left(n+\frac{1}{2}\right) U\right)
$$

and

$$
\begin{aligned}
Z_{n}^{\text {out }}= & \operatorname{det}\left(\frac{\Theta[\varrho]\left(Q_{-\beta}-Q_{+\alpha}+\left(n+\frac{1}{2}\right) U\right)}{E\left(Q_{-\beta}, Q_{+\alpha}\right)}\right) \Theta^{-l}[\varrho]\left(\left(n+\frac{1}{2}\right) U\right) \\
& \times \prod_{\alpha \neq \beta}\left(\frac{E\left(Q_{+\alpha}, Q_{+\beta}\right) E\left(Q_{-\alpha}, Q_{-\beta}\right)}{E^{2}\left(Q_{+\alpha}, Q_{-\beta}\right)}\right)^{-n-1 / 2} \prod_{\alpha} E^{2 n+1}\left(Q_{+\alpha}, Q_{-\alpha}\right) \\
= & \frac{\Theta[\varrho]\left(\left(n-\frac{1}{2}\right) U\right)}{\Theta[\varrho]\left(\left(n+\frac{1}{2}\right) U\right)} \prod_{i<j} E^{2 p_{i} p_{j} n}\left(Q_{i}, Q_{j}\right) .
\end{aligned}
$$

To obtain the last identity in (2.20) Corollary 2.19 from [12] was used. Note that the structure of $Z_{n}$ is the same as in the two-point case, so the same regularisation can be applied. Moreover, the regularized partition function $Z_{F}$ $=\Theta[\varrho](0)$ is the same as in the two-point case. The dependence on $Q_{i}$ is dropped in a natural way (providing $D=26$, so all the anomalies are cancelled).

The amplitude

$$
A_{F}=\left(Z_{1 / 2}^{\text {out }}\right)^{-1}=\frac{\Theta[\varrho](U)}{\Theta[\varrho](0)} \prod_{i<j} E^{p_{i} p_{j}}\left(Q_{i}, Q_{j}\right)
$$

The subscript $F$ denotes the amplitude is fermionic, and it is what we really have. 
In [4] Krichever proposed to obtain the bosonic vacuum expectation by averaging the fermionic expectation over all the $\varrho$-representations. The proposal was backed with the explicit calculation of the stress-energy tensor $T(z)$ in a case $g=1$. Here we shall prove the similar identity for the amplitudes:

\section{Lemma 2.}

$$
\log \left(A_{B}\right)=\left\langle\log \left(\left|A_{F}\right|^{2}\right)\right\rangle_{\varrho}=\int_{0}^{1} \ldots \int_{0}^{1} d^{2 g} \varrho \log \left|A_{F}\right|^{2} .
$$

Proof. Averaging the right-hand side we only need to find $\operatorname{Re}\left\langle\log \left(\frac{\Theta[\varrho](U)}{\Theta[\varrho](0)}\right)\right\rangle$. In Appendix B it is shown that

Hence,

$$
\operatorname{Re}\left\langle\log \left(\frac{\Theta[\varrho](U)}{\Theta[\varrho](0)}\right)\right\rangle=\pi \operatorname{Im} U^{t} \operatorname{Im} \tau^{-1} \operatorname{Im} U .
$$

where

$$
\exp \left\langle\log \left(\left|A_{F}\right|^{2}\right)\right\rangle_{\ell}=\prod_{i<j}\left|\Psi\left(Q_{i}, Q_{j}\right)\right|^{2 p_{i} p_{j}},
$$

$$
\Psi\left(Q_{i}, Q_{j}\right)=E\left(Q_{i}, Q_{j}\right) \exp \left(-\pi \operatorname{Im}\left(Q_{i}-Q_{j}\right)_{a}\left(\operatorname{Im} \tau^{-1}\right)_{a b} \operatorname{Im}\left(Q_{i}-Q_{j}\right)_{b}\right) .
$$

It is the well known generalised Kobo-Nielsen bosonic amplitude.

The lemma is proven.

It should be mentioned here that in our approach the amplitude is a tensor. At every point $Q_{i}$ in the local coordinates it has the tensor weight $p_{i}^{2}$. That is the usual relation $\Delta=p^{2}$ of the Veneziano model. Fix now the Euclidean time coordinates $\xi_{i}$ (2.17) and write:

$$
\prod_{i}\left|d \xi_{i}\right|_{i}^{p_{i}^{2}}=\prod_{i<j}\left|E\left(Q_{i}, Q_{j}\right)\right|^{p_{i} p_{j}} \exp \left(-2 \pi \operatorname{Im} U^{t} \operatorname{Im} \tau^{-1} \operatorname{Im} U\right) \prod\left|d z_{i}\right|^{p_{i}^{2}} .
$$

Hence, the bosonic amplitude has a simple geometric implication - it is a Jacobian that transforms the form $\prod\left|d z_{i}\right|^{p_{i}^{2}}$ to the free strings coordinates. Particularly, in the euclidean time coordinates it is one.

Theorem 2 is proven.

The remarkable identity (2.24) was found by Krichever for the genus 0 case. $\mathrm{He}$ also suggested it should be true for arbitrary genus.

\section{Appendix A}

In this paper we deal with a Riemann surface $\Gamma$ of genus $g$. The basis of 1-cycles in $H_{1}(\Gamma)$ is $a_{i}, b_{i}, i=1, \ldots, g$ and normalised:

$$
a_{i} \circ a_{j}=b_{i} \circ b_{j}=0, \quad a_{i} \circ b_{j}=\delta_{i j} .
$$

On $\Gamma$ live $g$ holomorphic 1-differentials $\omega_{i}(z)$, dual to 1-cycles:

$$
\oint_{a_{i}} \omega_{j}(z)=\delta_{i j} \oint_{b_{i}} \omega_{j}(z)=\tau_{i j} .
$$


As usual, the period matrix $\tau$ defines Riemann theta-function (with the characteristics $\varepsilon, \delta$ ):

$$
\Theta\left[\begin{array}{l}
\varepsilon \\
\delta
\end{array}\right](z)=\sum_{n \in Z^{g}} \exp \left(\pi i(n+\varepsilon)^{T} \tau(n+\varepsilon)+2 \pi i(z+\delta)^{T}(n+\varepsilon)\right) z \in C^{g} .
$$

The Abelian map $A: z \rightarrow A(z)=\left(\int_{P}^{z} \omega_{1}, \ldots, \int_{P}^{z} \omega_{g}\right)$ sets the correspondence $\Gamma \rightarrow C^{g} / Z^{g} \otimes \tau Z^{g}=\operatorname{Jac}(\Gamma)$. From now on we denote the point $z$ on $\Gamma$ and its image $A(z)$ on $\operatorname{Jac}(\Gamma)$ by the same symbol $z$ if it does not lead to confusion.

There are two special objects on $\Gamma$, useful for us. The prime-form $E(z, w)$ is a $-\frac{1}{2}$-differential both on $z$ and $w$,

Near the diagonal $z=w$

$$
E(z, w)=-E(w, z) .
$$

$$
E(z, w)=(z-w)(1+o(z)) d z^{-1 / 2} d w^{-1 / 2}
$$

and

Fay's $\sigma$-differential

$$
E(z, w)=0 \Leftrightarrow z=w .
$$

$$
\sigma(z)=\frac{\Theta(z-g P-\Delta)}{E^{g}(z, P)}
$$

( $\Delta$ stands for the Riemann class) has tensor weight $g / 2$. It does not (up a factor, independent on $z$ ) depend on the auxilliary point $P$, holomorphic on $\Gamma$ and has no zeroes.

For a detailed review on Riemann surfaces see [12].

\section{Appendix B}

We prove here Lemma 2. All one needs is to compute the average

$$
\operatorname{Re}\left\langle\log \frac{\Theta[\varrho](z)}{\Theta[\varrho](0)}\right\rangle_{\varrho} .
$$

To find it, let us make use of the theta-functional transformational properties:

$$
\Theta\left[\begin{array}{l}
\varepsilon \\
\delta
\end{array}\right](z+\tau M+N)=\exp \left(-\pi i M^{t} \tau M-2 \pi i M^{t}(z+N) \Theta\left[\begin{array}{l}
\varepsilon \\
\delta
\end{array}\right](z) .\right.
$$

In particular, it shows that $\Theta(z)$ is periodic in $\operatorname{Re} z$.

First, by definition, one has

$$
\begin{aligned}
\operatorname{Re}\left\langle\log \Theta\left[\begin{array}{l}
\varepsilon \\
\delta
\end{array}\right](z)\right\rangle= & -\pi\left\langle\varepsilon^{t} \operatorname{Im} \tau \varepsilon-2 \pi \varepsilon^{t} \operatorname{Im} z\right\rangle \\
& +\operatorname{Re}\langle\log \Theta(z+\tau \varepsilon+\delta)\rangle
\end{aligned}
$$


The second term is

$$
\begin{aligned}
\operatorname{Re} & \int_{0}^{1} \ldots \int_{0}^{1} d^{g} \varepsilon d^{g} \delta \log \Theta(z+\tau \varepsilon+\delta) \\
& =\operatorname{Re} \int_{0}^{1} \ldots \int_{0}^{1} d^{g} \varepsilon d^{g} \delta \log \Theta\left(\tau\left(\varepsilon+(\operatorname{Im} \tau)^{-1} \operatorname{Im} z\right)+\delta\right) .
\end{aligned}
$$

Let us denote $(\operatorname{Im} \tau)^{-1}$ by $y=\left(y_{1}, \ldots, y_{g}\right)$ and the shifted arguments

Then

$$
\begin{gathered}
\varepsilon+\left(y_{1}, 0, \ldots, 0\right)=\varepsilon^{\prime}, \\
\left(0, y_{2}, \ldots, y_{g}\right)=y^{\prime}
\end{gathered}
$$

$$
\begin{aligned}
\operatorname{Re} \int_{0}^{1} \ldots \int_{0}^{1} d^{g} \varepsilon d^{g} \delta \log \Theta(z+\tau \varepsilon+\delta) \\
=\operatorname{Re} \int_{y_{1}}^{1+y_{1}} \ldots \int_{0}^{1} d^{g} \varepsilon^{\prime} d^{g} \delta \log \Theta\left(\tau\left(\varepsilon^{\prime}+y^{\prime}\right)+\delta\right) \\
=\operatorname{Re} \int_{0}^{1} \ldots \int_{0}^{1} d^{g} \varepsilon^{\prime} d^{g} \delta \log \Theta\left(\tau\left(\varepsilon^{\prime}+y^{\prime}\right)+\delta\right) \\
\quad+\operatorname{Re} \int_{0}^{y_{1}} \ldots \int_{0}^{1} d^{g} \varepsilon d^{g} \delta\left(-\pi i \tau_{11}-2 \pi i\left\{\sum_{i=1}^{g} \tau_{1 i}\left(\varepsilon_{i}+y_{i}\right)+\delta \delta_{1}\right\}\right) \\
=\operatorname{Re}\left\langle\log \Theta\left(\tau\left(\varepsilon+y^{\prime}\right)+\delta\right)\right\rangle \\
\quad+\pi \operatorname{Im} \tau_{11} y_{1}+2 \pi \sum_{i=2}^{g}\left(\operatorname{Im} \tau_{l i}\right)\left(\frac{y_{1}}{2}+y_{i} y_{1}\right)+2 \pi \operatorname{Im} \tau_{11} \frac{y_{1}^{2}}{2} \\
=(\operatorname{after} g \operatorname{steps}) \ldots \\
=\operatorname{Re}\langle\log \Theta(\tau \varepsilon+\delta)\rangle+\pi \sum_{i, j=1}^{g}\left(1+y_{i}\right) y_{j}\left(\operatorname{Im} \tau_{i j}\right)+\text { const. }
\end{aligned}
$$

Adding the average of the first term in the right-hand side of (B.2) we get:

$$
\begin{aligned}
\operatorname{Re}\left\langle\log \frac{\Theta\left[\begin{array}{l}
\varepsilon \\
\delta
\end{array}\right](z)}{\Theta\left[\begin{array}{l}
\varepsilon \\
\delta
\end{array}\right](0)}\right\rangle & =\pi \sum_{i, j=1}^{g}\left(1+y_{i}\right) y_{j}\left(\operatorname{Im} \tau_{i j}\right)-\pi \sum_{i=1}^{g} \operatorname{Im} z_{i} \\
& =\pi \sum_{i, j=1}^{g}\left(\operatorname{Im} z_{i}\right)\left(\operatorname{Im} z_{j}\right)(\operatorname{Im} \tau)_{i j}^{-1}
\end{aligned}
$$

Lemma 2 is proven.

Acknowledgements. I thank S. P. Novikov for the support and valuable discussions. I am grateful also to I. M. Krichever, for his comments on the soliton theory technique. 


\section{References}

1. Krichever, I.M., Novikov, S.P.: Virasoro-type algebras, Riemann surfaces and structures of the soliton theory. Func. Anal. Pril. 1, 46-63 (1987)

2. Krichever, I.M., Novikov, S.P.: Virasoro-type algebras, Riemann surfaces and string in Minkovski space. Func. Anal. Pril. 214, 47-61 (1987)

3. Krichever, I.M., Novikov, S.P.: Virasoro-type algebras, pseudo-tensor of energy-momentum and operator expansions on the Riemann surfaces. Func. Anal. Pril. 231 (1989)

4. Krichever, I.M., Novikov, S.P.: Riemann surfaces, operator fields, strings. Analogues of the Fourier-Laurent bases. Preprint IHES/P/89/26 (April 1989)

5. Krichever, I.M.: Spectral theory of two-dimensional periodic operators and its applications. Uspec. Mat. Nauk. 442, 121-183 (1989)

6. Krichever, I.M.: The algebraic curves and the nonlinear equations. Uspec. Mat. Nauk. 334, 215-216 (1978)

7. Polyakov, A.M.: Phys. Lett. 13, 207 (1981);

Belavin, A., Knizhnik, V.: Phys. Lett. 168 B, 201 (1986);

Morozov, A.Yu.: Phys. Lett. 184 B, 171 (1987)

8. Dick, R.: Lett. Math. Phys. 18, 255 (1989);

Dick, R.: Holomorphic differentials on Punctured Riemann surfaces. Preprint DESY 89-097 (August 1989)

9. Schlichenmaier, M.: Central extensions and semi-infinite wedge representations of KricheverNovikov algebras for more than two points. Preprint Manusk. Fak. Math. u. Inf. Mannheim 102-1989 (September 1989)

10. Jaffe, A., Klimek, S., Lesniewski, A.: Representations of the Heisenberg algebra on a Riemann surface. Preprint HUTMP 89/B239 (April 1989)

11. Venetziano, S.: An introduction to dual models. Phys. Rep. 9, 199 (1974)

Green, M.B., Schwarz, J.H., Witten, E.: Superstring theory I, II. Cambridge: Cambridge University Press 1987

12. Fay, J.: Theta functions on Riemann surfaces. Lecture Notes in Mathematics, vol. 352. Berlin, Heidelberg, New York: Springer 1973

Communicated by Ya. G. Sinai 
